\title{
On the electron temperature downstream of the solar wind termination shock
}

\author{
I. V. Chashei ${ }^{1}$ and H. J. Fahr ${ }^{2}$ \\ ${ }^{1}$ Lebedev Physical Institute, Leninskii Prospect 53, 117924 Moscow, Russian Federation \\ ${ }^{2}$ Argelander Institut für Astronomie, Astrophysics Department, University of Bonn, Auf dem Huegel 71, 53121 Bonn, \\ Germany
}

Correspondence to: H. J. Fahr (hfahr@astro.uni-bonn.de)

Received: 29 January 2013 - Revised: 15 April 2013 - Accepted: 5 June 2013 - Published: 9 July 2013

\begin{abstract}
In this paper we study the temperatures of electrons convected with the solar wind to large solar distances and finally transported over the solar wind termination shock. Nearly nothing, unless at high energies in the cosmic ray regime, is known about the thermodynamical behaviour of these distant electrons from in situ plasma observations. Hence it is tacitly assumed these electrons, due to their adiabatic behaviour and vanishing heat conduction or energization processes, have rapidly cooled off to very low temperatures once they eventually arrive at the solar wind termination shock (at about $100 \mathrm{AU}$ ). In this paper we show that such electrons, however, at their passage over the termination shock due to the shock-electric field action undergo an overadiabatic heating and therefore appear on the downstream side as a substantially heated plasma species. Looking quantitatively into this heating process we find that solar wind electrons achieve temperatures of the order of $2-4 \times 10^{6} \mathrm{~K}$ downstream of the termination shock, depending on the upstream solar wind bulk velocity and the shock compression ratio. Hence these electrons therewith play an important dynamical role in structuring this shock and determining the downstream plasma flow properties. Furthermore, they present an additional ionization source for incoming neutral interstellar hydrogen and excite X-ray emission. They also behave similar to cosmic ray electrons and extend to some limited region upstream of the shock of the order of $0.1 \mathrm{AU}$ by spatial diffusion and thereby also modify the upstream solar wind properties.
\end{abstract}

Keywords. Interplanetary physics (Interplanetary shocks; Solar wind plasma) - Space plasma physics (Electrostatic structures)

\section{Introduction}

The theoretical description of the plasma passage over an astrophysical MHD (magnetohydrodynamics) shock, e.g. the solar wind termination shock, even nowadays is not a generally agreed upon topic. Especially the role of electrons in structuring such a shock is not at all well understood, though, as it appears to us, this role is extremely important. Usually it is assumed that ions and electrons remain in thermodynamical equilibrium at the shock passage, thus retaining their pre-shock densities and temperatures with identical, though changed, densities and pressures also downstream of the shock. It has, however, meanwhile been recognized that electrons are likely to be heated over-adiabatically (see Leroy et al., 1982; Sgro and Nielson, 1976; Schwartz et al., 1988; or Tokar et al., 1986). This points to the fact that non-adiabatic heating, at least of electrons, takes place at the shock, which seems to be controlled by the conservation of the magnetic particle moment (Schwartz et al., 1988).

The so-called "magnetic moment" of charged particles, $\mu=m v_{\perp}^{2} / 2 B$, has experienced a somewhat controversial appreciation in the plasma literature of the past. Though in modern MHD shock simulations of the solar wind termination shock it is not explicitly required that ions and electrons conserve their magnetic moment, there are, on the other hand, affirmative conclusions achieved in the earlier shock literature. For example one finds in the paper by Sarris and Van Allen (1974) that these authors using momentconserving theoretical calculations can successfully represent observational data obtained by the satellites Explorer 33 and 35 on shock-processed ions near Earth's bowshock. They show that a fairly good fit to the observational data is 
achieved when considering that these particles are multiply reflected at their magnetic mirror points and eventually move downstream of the shock with an energy gain corresponding to $v_{2}^{2} / v_{1}^{2} \simeq B_{2} / B_{1}$ (indices denote upstream (1) and downstream (2) values of particle velocity and magnetic field magnitudes, respectively). This result clearly proves that the magnetic moments of these particles are conserved while crossing over the shock.

Terasawa (1979) compares ion spectra of MHD shockreflected ions calculated by two different approaches, namely the "adiabatic approach" and the so-called "kink approach". The first is applicable when the shock structure is large with respect to the ion gyroradius; the second is applicable when the shock structure is small with respect to the ion gyroradius. Interestingly enough the results for the spectra of the reflected ions are nearly identical in both cases, emphasizing that obviously in both cases particles behave as if their magnetic moments are conserved, independent of the extent of the shock transition region.

A further hint is also given by Fahr and Siewert (2013) who show that the Liouville operator in conventional ion transport equations just takes care that particle energy and magnetic moment are conserved at free ion motions, while simultaneously operating typical Fokker-Planck diffusion terms, e.g. for pitch-angle scattering, can be additionally considered as counteracting the magnetic moment conservation. Nevertheless the full transport equation requires the operation of a Liouville operator describing the tendency to conserve particle invariants in the case of absence or weakness of stochastic processes. In this paper we shall therefore not only consider shocks that conserve magnetic particle moments and thereby strongly heat particles, but also pay attention to the very different influence this has on electrons compared to ions, as we shall show.

Another encouragement for proceeding in this direction is that Voyager-2 measurements at the termination shock (TS) crossing (Richardson et al., 2008) deliver data which are unpredicted by presently existing TS models in the literature, like those by Fahr and Chalov (2008) or Wu et al. (2009). Based on identical upstream plasma conditions, none of the presently existing models can consistently explain all the TS observations such as the compression ratio $s$ at the shock, the thermal proton temperature jump $T_{\mathrm{p}, 2} / T_{\mathrm{p}, 1}$, and the downstream magnetosonic Mach number of thermal protons. In this paper, we shall, however, show that with the shockheated electrons treated here all these measurements can be surprisingly well explained. It is important to take into account that the thermodynamical behaviour of thermal protons at the passage over the TS shock is essentially different from that of electrons, so that especially the downstream temperatures of these species are substantially different, i.e. the electrons are much hotter and even start spatially diffusing with respect to the background plasma flow as described by Ferreira et al. (2001a, b), Langner et al. (2001), Lange et al. (2006), Nkosi et al. (2011), or Potgieter and Nndanganeni (2013).

\section{The centre of mass system downstream of the shock}

Here we first want to find the electric potential jump that is responsible for braking down the upstream to the downstream bulk velocity. Looking at the shock-associated, inherent electric potential jump $\Delta \Phi$, it then can be derived that bulk velocities of protons and electrons, respectively, change according to

$e \Delta \Phi=\frac{1}{2} M\left(U_{1}^{2}-U_{\mathrm{p} 2}^{2}\right)$

and

$-e \Delta \Phi=\frac{1}{2} m\left(U_{1}^{2}-U_{\mathrm{e} 2}^{2}\right)$.

Here, $m$ and $M$ are electron and proton masses, and the suffixes " 1 " and " 2 " characterize upstream and downstream quantities.

Their resulting downstream momentum flows are thus given by

$F_{\mathrm{e} 2}=m n_{\mathrm{e} 2} U_{\mathrm{e} 2}^{2}$

and

$F_{\mathrm{p} 2}=M n_{\mathrm{p} 2} U_{\mathrm{p} 2}^{2}$.

Looking now for that bulk velocity of the centre of mass (COM) system, which results from these two momentum flows and represents the system to which the magnetic field is frozen-in, one then derives the following relation:

$F_{2}^{*}=M n_{\mathrm{p} 2} U_{\mathrm{p} 2}^{2}+m n_{\mathrm{e} 2} U_{\mathrm{e} 2}^{2}=2 n_{2}^{*}\left(\frac{m+M}{2}\right) U_{2}^{* 2}$.

Since in all systems the particle number flow must be conserved, one finds

$2 n_{1} U_{1}=2 n_{2}^{*} U_{2}^{*}=n_{\mathrm{e} 2} U_{\mathrm{e} 2}+n_{\mathrm{p} 2} U_{\mathrm{p} 2}$,

which yields

$M U_{\mathrm{p} 2}+m U_{\mathrm{e} 2}=2\left(\frac{m+M}{2}\right) U_{2}^{*}$.

This then delivers the bulk velocity of the centre of mass system, the COM-velocity, in the form

$U_{2}^{*}=\frac{1}{m+M}\left(M U_{\mathrm{p} 2}+m U_{\mathrm{e} 2}\right)$.

Inserting from Eq. (2) the above result for $U_{\mathrm{e} 2}$, one then finally obtains

$$
\begin{aligned}
U_{2}^{*} & =\frac{M U_{\mathrm{p} 2}+m \sqrt{\frac{M}{m}\left(U_{1 \mathrm{p}}^{2}-U_{2 \mathrm{p}}^{2}\right)}}{m+M} \\
& \simeq U_{2 \mathrm{p}}\left(1+\sqrt{\frac{s_{\mathrm{p}}^{2}-1}{1840}}\right)
\end{aligned}
$$


which clearly shows that the COM velocity is essentially identical to $U_{2, \mathrm{p}}$.

\section{The electron overshooting at the shock}

Fahr et al. (2012) have shown that the electrically-induced electron overshooting at the shock into the downstream COM plasma frame is a highly important physical process that eventually leads to strong electron heating, to entropy generation and to high compression ratios. Starting from the above consideration of a shock potential step $\Delta \Phi$ affiliated with the deceleration of the proton bulk velocity from its upstream to its downstream COM value, one finds that electrons in the first step from just this potential jump experience a strong bulk velocity acceleration, as they are injected into the downstream flow.

As Fahr et al. (2012) have shown, the initial overshoot velocity of the electrons (i.e. differential velocity with respect to the downstream COM flow) is given by

$\delta U_{\mathrm{e}}=\mid U_{2}-U_{1} \sqrt{1+\frac{M}{m}\left(1-\frac{1}{s^{2}}\right)}$,

where $U_{1,2}$ are the centre of mass plasma bulk velocities at the upstream and downstream side of the shock, respectively.

This formula evidently shows that according to the mass ratio $m / M=\mu_{\mathrm{e}, \mathrm{i}}=1 / 1840$ the resulting overshoot velocity turns out to be as large as

$$
\begin{aligned}
\delta U_{\mathrm{e}} & =\left|U_{2}-U_{1} \sqrt{1+\frac{M}{m}\left(1-\frac{1}{s^{2}}\right)}\right| \\
& \simeq\left(U_{1} / s\right)\left|1-\sqrt{\frac{M}{m}\left(s^{2}-1\right)}\right| \geq 43 U_{1} .
\end{aligned}
$$

\section{Conversion of overshoot kinetic into thermal energy}

The electrons overshooting from the upstream into the downstream COM system initially act like an electron jet. This jet, however, creates an unstable plasma condition, exciting electrostatic plasma waves that finally convert the overshoot kinetic energy into thermal energy. Hence, we now consider the Buneman instability in the downstream plasma frame of electrons moving with high speeds relative to protons, i.e. the COM bulk system, thereby acting as an instability driver.

The downstream electron overshoot speed is equal to

$$
U_{2 \mathrm{e}}=U_{2 \mathrm{p}} s\left\{1+(M / m)\left[1-\left(1 / s^{2}\right)\right]\right\}^{1 / 2}
$$

and the differential speed $\Delta U_{2}$ of electrons relative to protons is equal to

$$
\begin{aligned}
\Delta U_{2}=U_{2 \mathrm{e}}-U_{2 \mathrm{p}}=U_{2 \mathrm{p}}\left\{s \left[1+\frac{M}{m}[1\right.\right. & \left.\left.\left.-\frac{1}{s^{2}}\right]\right]^{1 / 2}-1\right\} \\
& \simeq U_{2 p} s \sqrt{\frac{M}{m}} .
\end{aligned}
$$

$\Delta U_{2}$ in most cases is much higher than thermal speeds of both the electrons, $v_{\text {the }}$, and the protons, $v_{\text {thp }}$, hence fulfilling the relation

$\Delta U_{2}>v_{\text {the }}>>v_{\text {thp }}$.

These jump-induced conditions are similar to those of a plasma with an electric current, in which the Buneman instability is triggered (Alexandrov et al., 1984; Chen, 1984). Due to the above inequalities at the initial stage of the instability, the approximation of a cold plasma is valid at least in the foot region of the shock. In this case the dispersion equation corresponding to the Buneman instability can be written in the following form (Alexandrov et al., 1984)

$k_{\mathrm{x}}^{2} \epsilon_{\perp}+k_{\mathrm{z}}^{2} \epsilon_{\|}=0$,

where $\epsilon_{\perp}$ and $\epsilon_{\|}$are the components of the cold plasma dielectric tensor with

$\epsilon_{\perp}=1-\sum \frac{\omega_{\mathrm{pa}}^{2}}{\left[\left(\omega-\mathbf{k} \cdot \mathbf{u}_{\mathrm{a}}\right)^{2}-\omega_{\mathrm{Ba}}^{2}\right]}$

and

$\epsilon_{\|}=1-\sum \frac{\omega_{\mathrm{pa}}^{2}}{\left(\omega-k u_{\mathrm{a}}\right)^{2}}$.

The $\alpha$-indices correspond to electrons and protons, and $\omega_{\mathrm{pa}}$ and $\omega_{\mathrm{Ba}}$ are plasma frequencies and cyclotron frequencies, respectively. In the reference frame moving with protons we find from Eqs. (15) and (16) the following dispersion equation:

$$
\begin{gathered}
\omega_{\mathrm{pe}}^{2} \cos ^{2} \theta\left[\left(\omega-\mathbf{k} \cdot \Delta \mathbf{U}_{2}\right)^{-2}+\frac{m}{M} \omega^{-2}\right] \\
+\omega_{\mathrm{pe}}^{2} \sin ^{2} \theta\left\{\left[\left(\omega-\mathbf{k} \cdot \Delta \mathbf{U}_{2}\right)^{2}-\omega_{\mathrm{Be}}^{2}\right]^{-1}\right. \\
\left.+\frac{m}{M}\left[\omega^{2}-\omega_{\mathrm{Bi}}^{2}\right]^{-1}\right\}=1 .
\end{gathered}
$$

We now consider the dispersion Eq. (17) at the condition of maximal growth of the disturbance, i.e. at $\omega \ll \mathbf{k} \cdot \Delta \mathbf{U}_{2} \approx$ $\omega_{\text {pe }}$; the inequality corresponds to the a-periodic type of instability and the equality corresponds to the resonant condition. We will take into account that in the solar wind plasma $\omega_{\mathrm{pe}}^{2} \gg \omega_{\mathrm{Be}}^{2}$ (i.e. $c \gg v_{\mathrm{A}} \sqrt{M / m}$ ) is always valid. Indeed, the ratio $\omega_{\mathrm{pe}}^{2} / \omega_{\mathrm{Be}}^{2}=(m / M)\left(c^{2} / v_{\mathrm{A}}^{2}\right)$ for typical solar wind plasma parameters is sufficiently high, i.e. $\omega_{\mathrm{pe}}^{2} / \omega_{\mathrm{Be}}^{2}>10^{4}$. Also note that in the distant solar wind at $r>5 \mathrm{AU}$ the ratio $\omega_{\mathrm{pe}}^{2} / \omega_{\mathrm{Be}}^{2}$ does not depend on solar distance. Assuming also $\omega / \omega_{\mathrm{Bi}} \gg 1$, one can show that Eq. (17) has three solutions,

$\omega=(m / 2 M)^{1 / 3} \omega_{\text {pe }}=\omega_{1}$,
$\omega=\omega_{2,3}=(1 / 2)(1 \pm i \sqrt{3}) \omega_{1}$,

i.e. under conditions of the distant solar wind the influence of the local magnetic field can be neglected. The Buneman 
instability has a strong growth rate which is maximal at the resonance condition, $\boldsymbol{k} \cdot \boldsymbol{\Delta} \boldsymbol{U}_{2}=\omega_{\text {pe }}$ (see Alexandrov et al., 1984; Chen, 1984), and there it is given by

$\gamma_{\max }=\sqrt{\frac{3}{4}}(m / 2 M)^{1 / 3} \omega_{\mathrm{pe}}$

Note that the inequality $\omega / \omega_{\mathrm{Bi}} \approx(M / m)^{2 / 3}\left(\omega_{\mathrm{pe}} / \omega_{\mathrm{Be}}\right) \gg 1$ used above is fulfilled for Eq. (20). The typical growth period $\gamma_{\max }$ of this instability is of the order of $10^{-4} \mathrm{~s}$ for typical values of the plasma density of $n \approx 10^{-3} \mathrm{~cm}^{-3}$ at solar distances of about $100 \mathrm{AU}$. Landau damping of these oscillations at protons is very weak under these conditions and can be neglected in our ongoing considerations.

The Landau damping at electrons is described by the decrement (Alexandrov et al., 1984; Chen, 1984)

$\gamma_{\text {Le }}=\sqrt{\frac{\pi}{8}} \omega_{\text {pe }}\left(\frac{\omega_{\text {pe }}}{k_{\|} v_{\text {the }}}\right)^{3} \exp \left[-1.5-\left(\omega_{\text {pe }} / k_{\|} v_{\text {the }}\right)^{2}\right]$,

where $k_{\|}$is the component of the wavevector $\boldsymbol{k}$ parallel to the magnetic field. Taking into account the condition $\Delta U_{2}>$ $v_{\text {the }} \gg v_{\text {thp }}$, one can see that, at the initial stage of the instability, Landau damping is exponentially weak. This is the reason why the nonlinear stage of the instability develops very fast and leads to plasma stratifications on very small scales, i.e. scales of the order $l_{\mathrm{S}} \approx \Delta U_{2} / \omega_{\mathrm{pe}}$. An interaction between electron plasma bunches and oscillating electric fields then results in electron braking and proton acceleration. During this process the relative speed $\Delta U_{2}$ will decrease and, in line with this, Landau damping given in Eq. (21) becomes more and more important. This finally leads to electron heating and, in turn, to further deceleration and heating of electrons.

Such a scenario can be called a spontaneous local relaxation. It occurs if the shock front can be considered as infinitesimally thin. In the opposite case of an extended shock front structure, however, a spatial scenario takes place in which the relaxation to the stable state is instead a continuous process acting at the instability threshold $\gamma_{\max }=\gamma_{\mathrm{Le}}$ along the whole effective length of the shock-induced electric potential jump. The full electron speed $U_{2 \mathrm{e}}$ is not fully achieved in this spatial scenario because the work of the electric potential on electrons is continuously transferred to electron heating. This is a little similar to the case already considered by Verscharen and Fahr (2008) for the parallel MHD shock. The final stage of the instability for both scenarios is the motion of protons and heated electrons with one and the same speed $U_{2}^{*}$ equal to the downstream bulk speed but with electrons that appear much more heated than at classical Rankine-Hugoniot shocks.

In this paper we only consider the Bunemann instability and show that under cold plasma conditions and low electron beta conditions this instability can do the job to efficiently thermalize the electron overshoot kinetic into electron thermal energy. In favour of this process operating, one should consider the foot region of the shock where the background plasma can be considered as sufficiently cold. As shown by Scholer and Matsukiyo (2004), competing with the Bunemann instability the modified two-stream instability should be considered as heating electrons. The relative efficiency of these two instabilities is strongly related to the Mach number of the shock and of the upstream electron plasma beta value. For electron plasma beta values below 0.02 , which as we believe characterizes the upstream conditions at the termination shock, Bunemann instability should, however, be dominant.

In fact, in principle one also has to consider energy appearing in waves and turbulences that are driven by these instabilities. This we cannot do in this paper in a consistent form. Thus we have to conclude here that the thermal energy, according to our present calculation created by electron thermalization, somehow represents an upper limit of what can be discussed in this context.

\section{The electron temperature jump at the shock}

Now we evaluate as function of the downstream COM plasma speed the fraction of relative kinetic energy transferred to thermal energy of the electrons. We had obtained the COM bulk speed which with $m \ll M$ leads to

$$
U_{2} \approx U_{2 \mathrm{p}}+(m / M) U_{2 \mathrm{e}} \approx U_{2 \mathrm{p}}+s \sqrt{m / M} U_{2 \mathrm{p}}
$$

and shows that the difference between $U_{2}$ and $U_{2 \mathrm{p}}$ is small in comparison with $U_{2 \mathrm{p}}$.

The part $\Delta W_{\mathrm{e}}$ of electron kinetic energy converted into heat can then be found from the energy conservation law applied to the pair of particles thusly:

$2 \Delta W_{\mathrm{e}}=m U_{2 \mathrm{e}}^{2}+M U_{2 \mathrm{p}}^{2}-(m+M) U_{2}^{2}$,

which results in

$2 \Delta W_{\mathrm{e}}=\left[\frac{m M}{m+M}\right]\left(U_{2 \mathrm{e}}-U_{2 \mathrm{p}}\right)^{2}$

or

$\Delta W_{\mathrm{e}} \approx(1 / 2) m U_{2 \mathrm{e}}^{2}$.

This expresses the fact that essentially the whole kinetic energy of the overshooting electrons is converted into electron heat, meaning that the speed $U_{2 \mathrm{e}}$ plays the role of the downstream thermal speed of heated electrons.

Hence the above relation allows one to estimate the jump in the electron temperature $\Delta T_{1 \mathrm{e}}$ after shock passage by the following expression

$$
\begin{aligned}
\Delta T_{1 \mathrm{e}} & =m U_{2 \mathrm{e}}^{2} / 3 k=\left(m \Delta U_{1}^{2} / 3 k\right)\left\{1+\frac{M}{m}\left[1-\left(1 / s^{2}\right)\right]\right\} \\
& \approx\left(M \Delta U_{1}^{2} / 3 k\right)\left[1-\left(1 / s^{2}\right)\right] .
\end{aligned}
$$


Using typical values for the termination shock (see Richardson et al., 2008) with $s \approx 2.5$ and $\Delta U_{1} \approx 350 \mathrm{~km} \mathrm{~s}^{-1}$, we find from the above relation

$\Delta T_{1 \mathrm{e}} \approx M \Delta U_{1}^{2} / 18 k \approx 3.5 \times 10^{6} \mathrm{~K}$.

Thus, surprisingly enough, the above shows that the electron temperature $T_{\mathrm{e}, 2}$ downstream of the shock must be expected to be much higher than the measured downstream solar wind proton temperature $T_{\mathrm{p}, 2}$, which is only of the order of a few $10^{5} \mathrm{~K}$, hence yielding ratios of $T_{\mathrm{e}, 2} / T_{\mathrm{p}, 2} \simeq 35$.

The above formula also shows that electrons, when propagating downstream of the shock, will finally have picked up about $(1 / 6)$ of the upstream kinetic proton energy with respect to the downstream bulk velocity rest frame, because from the above one finds

$3 / 2 k \Delta T_{1 \mathrm{e}} \approx(1 / 6)\left[\frac{1}{2} M \cdot \Delta U_{1}^{2}\right]$.

\section{Spatial diffusion of shock-processed electrons}

Another question of importance may now, however, occur connected with the new appearance of energetic downstream electrons, which is that by their new energy they may partly attain the freedom to spatially diffuse relative to the bulk flow of the background plasma, i.e. they might not any longer be co-convected with the solar wind bulk flow. The adequate transport equation for the electron pressure $P_{\mathrm{e}}$ is derived from the original transport equation for the cosmic ray distribution function (see Parker, 1965; Potgieter, 1996) and after neglect of curvature drifts and production of pressure moments (see e.g. Fichtner et al., 1996; Kissmann et al., 2003) in the case of electrons is given by the following (Ferreira et al., 2001a, b; Langner et al., 2001; Lange et al., 2006; Nkosi et al., 2011; Potgieter and Nndanganeni, 2013):

$$
\frac{\partial P_{\mathrm{e}}}{\partial t}=\nabla \cdot\left(\hat{\kappa} \cdot \nabla P_{\mathrm{e}}\right)-\boldsymbol{U} \cdot \nabla P_{\mathrm{e}}-\frac{4}{3} P_{\mathrm{e}}(\nabla \cdot \boldsymbol{U}),
$$

where $\hat{\kappa}$ denotes the spatial diffusion tensor for the electrons. Studying in-ecliptic regions where near the termination shock the Archimedean magnetic fields are quasi-azimuthal, this then means that a diffusive transport of electrons off the position of the shock in upstream direction mainly works by spatial diffusion perpendicular to the magnetic field, regulated by the tensor element $\kappa_{\perp}$. Thus the above vector equation for upstream electron diffusion then reduces to the following scalar equation:

$$
\begin{aligned}
\frac{\partial P_{\mathrm{e}}}{\partial t}= & \frac{1}{r^{2}} \frac{d}{d r}\left[r^{2}\left(\kappa_{\perp} \cdot \frac{d}{d r} P_{\mathrm{e}}\right)\right] \\
& -U \cdot \frac{d}{d r} P_{\mathrm{e}}-\frac{4}{3} P_{\mathrm{e}} \cdot \frac{d}{d r} U
\end{aligned}
$$

with $r$ being the solar distance. This then essentially means

$$
\frac{\partial P_{\mathrm{e}}}{\partial t} \simeq \frac{2}{r}\left(\kappa_{\perp} \frac{d}{d r} P_{\mathrm{e}}\right)+\frac{d}{d r}\left[\left(\kappa_{\perp} \frac{d}{d r} P_{\mathrm{e}}\right)-U P_{\mathrm{e}}\right]
$$

This shows that at large distances $\left(r / r_{0} \gg 1 ; r_{0}=r_{E}=\right.$ $1 \mathrm{AU})$ only the second term on the right-hand side counts and thus diffusion competes with convection if the following relation holds:

$\kappa_{\perp} \frac{d}{d r} P_{\mathrm{e}} \simeq U P_{\mathrm{e}}$.

At the termination shock this relation can be interpreted as stating that, at a critical value of a relative change in the electron pressure (or temperature), electron spatial diffusion starts competing with convection. This critical value can be calculated from the following relation:

$P_{\mathrm{e} 1}^{-1}\left|\frac{d}{d r} P_{\mathrm{e}}\right|_{\mathrm{TS}} \simeq \frac{\Delta P_{\mathrm{e}}}{P_{e_{1}}} \frac{1}{D} \simeq U_{1} / \kappa_{\perp}$,

where $D$ denotes the shock transition distance. This then leads to the following limit:

$\Delta P_{\mathrm{e}} \simeq \frac{P_{\mathrm{e} 1} D}{\kappa_{\perp}} U_{1}$,

or written in terms of temperature by

$\Delta T_{\mathrm{e}} \simeq \frac{n_{\mathrm{e} 1} T_{\mathrm{e} 1} D}{n_{\mathrm{e} 2} \kappa_{\perp}} U=\frac{D}{s \kappa_{\perp}} U_{1} T_{\mathrm{e} 1}$.

Using now the result that we have presented in the section above (see Eq. 26) will give us

$\Delta T_{1 \mathrm{e}}=\left[1-\left(1 / s^{2}\right)\right] \frac{M \Delta U_{1}^{2}}{3 k} \simeq \frac{D}{s \kappa_{\perp}} U_{1} T_{\mathrm{e} 1}$,

where $s$ denotes the shock compression ratio and leads to the requirement

$\left[1-\frac{1}{s^{2}}\right] \frac{M U_{1}^{2}\left(1-\frac{1}{s}\right)^{2}}{3 k} \simeq \frac{D}{s \kappa_{\perp}} U_{1} T_{\mathrm{e} 1}$,

which for the crucial extent $D_{\mathrm{c}}$ of the transition region means

$D_{\mathrm{c}} \simeq(s+1)\left(1-\frac{1}{s}\right)^{3} \frac{\frac{1}{2} M U_{1}^{2}}{\frac{3}{2} k T_{\mathrm{e} 1}} \frac{\kappa_{\perp}}{U_{1}}$.

Now we want to evaluate this expression and start with choosing

$\frac{1}{2} M U_{1}^{2} / \frac{3}{2} k T_{\mathrm{e} 1}=M_{s 1}^{2} \simeq 10^{2}$

and with setting $s=2.5$, which yields

$(s+1)\left(1-\frac{1}{s}\right)^{3}=0.75$.

This then finally leads to

$D_{\mathrm{c}} \simeq 75 \frac{\kappa_{\perp}}{U_{1}}$. 
From Lange et al. (2006) we take

$\kappa_{\perp}(r, v)=10^{-2} \cdot \kappa_{0} \cdot \beta(v) \cdot g(r, v)$,

where $\kappa_{0}=4.5 \times 10^{22} \mathrm{~cm}^{2} \mathrm{~s}^{-1}$ is a constant and $\beta(v)=v / c$. For a $1 \mathrm{MeV}$ electron, one thus has $\beta(\bar{v})=\bar{v} / c=0.86$. The function $g(r, v)$ in our case is given by (Ferreira et al., 2001a, b):

$g(r, v)=g\left(r_{\mathrm{s}}, \bar{v}\right)=(1 / 5)\left(P_{0} / P_{0}\right)^{0.6}(1 / 50)\left(\bar{P} / P_{0}\right)\left(r_{\mathrm{s}} / r_{0}\right)^{2}$,

where $P_{0}=1 \mathrm{GV}$ and $r_{\mathrm{s}}=100 r_{0}$. Thus for the function $f$ one obtains

$g\left(r_{\mathrm{s}}, \bar{v}\right)=(1 / 5)(1 / 50)\left(10^{-3}\right) \times(100)^{2}=4 \times 10^{-4}$.

Hence the diffusion coefficient is then calculated by

$$
\begin{aligned}
\kappa_{\perp}\left(r_{\mathrm{s}}, \bar{v}\right) & =10^{-2} \cdot 4.5 \times 10^{22} \cdot 0.86 \cdot 4 \times 10^{-4} \\
& =1.55 \times 10^{17} \cdot\left[\mathrm{cm}^{2} \mathrm{~s}^{-1}\right] .
\end{aligned}
$$

With the above values we then finally find

$$
\begin{aligned}
D_{\mathrm{c}} & \simeq 75 \frac{\kappa_{\perp}}{U_{1}}=75 \cdot 1.55 \times 10^{17} / 4.5 \times 10^{7} \\
& =2.58 \times 10^{11} \mathrm{~cm} \simeq 0.02 \mathrm{AU} .
\end{aligned}
$$

This says that $1 \mathrm{MeV}$ electrons appearing near the shock should be able to diffusively redistribute to a region of about a few $10^{-2}$ AU upstream of the shock over which they build up their strong temperature ramp. Considering that in the $10^{6} \mathrm{~K}$ electron velocity distribution function also higher than $1 \mathrm{MeV}$ energetic electrons are contained thus means that such higher energetic electrons can even be expected at $1 \mathrm{AU}$ upstream of the shock.

\section{Impact ionization by shock-processed electrons}

Finally, here we would like to briefly look at the electron impact ionization of neutral interstellar $\mathrm{H}$-atoms by these shock-processed electrons. As shown in Rucinski and Fahr (1989), the electron-impact ionization rate of $\mathrm{H}$-atoms is given by

$\beta_{\mathrm{e}, \mathrm{H}}(r)=\frac{8 \pi}{m_{\mathrm{e}}^{2}} \int_{E_{\mathrm{H}, \mathrm{i}}}^{\infty} \sigma_{\mathrm{H}}(E) f(E, r) E d E\left[\mathrm{~cm}^{3} \mathrm{~s}^{-1}\right]$,

where $m_{\mathrm{e}}$ is the electron mass, $E_{\mathrm{H}, \mathrm{i}}=13.6 \mathrm{eV}$ is the ionization energy of the $\mathrm{H}$-atom , $\sigma_{\mathrm{H}}(E)$ is the $\mathrm{H}$-atom impact ionization cross section, and $f(E, r)$ denotes the electron energy distribution function. With the impact cross section given by Lotz (1967) in the form

$$
\begin{aligned}
\sigma_{\mathrm{H}}(E)= & 5.77 \cdot\left[1-0.75 \exp \left(-0.46\left(E / E_{\mathrm{H}, \mathrm{i}}-1\right)\right)\right. \\
& \cdot \frac{2 \ln \left(E / E_{\mathrm{H}, \mathrm{i}}\right)}{E E_{\mathrm{H}, \mathrm{i}}}\left[10^{-14} \mathrm{~cm}^{2}\right],
\end{aligned}
$$

one is then lead to the following ionization rate when assuming the electron velocity distribution function to be a Maxwellian with a temperature $T_{\mathrm{e}, 2}$ (see Rucinski and Fahr, 1989):

$$
\begin{aligned}
\beta_{\mathrm{e}, \mathrm{H}}(r)= & 11.6 \times 10^{-14} \\
& \cdot\left[\frac{G}{\Phi} E i(-\Phi)-\frac{C}{\alpha} E i(-\alpha)\right]\left[\mathrm{cm}^{3} \mathrm{~s}^{-1}\right] .
\end{aligned}
$$

In the above the following definitions have been used:

$$
\begin{aligned}
& G=0.75 \exp (0.46) C \\
& C=n_{\mathrm{e}, 2}(r) \\
& \alpha=\frac{E_{\mathrm{H}, \mathrm{i}}}{K T_{\mathrm{e}, 2}} \\
& \Phi=\alpha+0.46 \\
& E i(-b)=-b \int^{\infty} \exp (-b x) \ln x d x .
\end{aligned}
$$

With the earlier result of shocked electrons representing a temperature of $K T_{\mathrm{e}, 2} \simeq 1 \mathrm{MeV}$ and having a downstream electron density of $n_{\mathrm{e}, 2}(r)=2 \times 10^{-3} \mathrm{~cm}^{-3}$, we then calculate with the following definitions:

$G=0.75 \exp (0.46) 2 \times 10^{-3}$

$C=2 \times 10^{-3}$

$\alpha=\frac{E_{\mathrm{H}, \mathrm{i}}}{K T_{\mathrm{e}, 2}}=\frac{13.6}{10^{6}}=1.36 \times 10^{-5}$

$\Phi=0.46$

$E i(-\alpha)=-1.36 \times 10^{-5} \int^{\infty} \exp \left(-1.36 \times 10^{-5} x\right) \ln x d x$,

obtaining the result

$\beta_{\mathrm{e}, \mathrm{H}}(r)=23.2 \times 10^{-17}$.

$\left[\frac{1.18}{0.46} E i(-0.46)+E i\left(-1.36 \times 10^{-5}\right)\right] \simeq 3.3 \times 10^{-9} \mathrm{~s}^{-1}$

Compared to the charge exchange frequency $\beta_{\mathrm{p}, \mathrm{ch}}=\sigma_{\mathrm{ex}}$. $n_{\mathrm{p}, 2} \cdot \bar{v}_{\mathrm{rel}, \mathrm{p}}=2 \times 10^{-11} \mathrm{~s}^{-1}$ with downstream solar wind protons, one thus obtains a ratio of

$\frac{\beta_{\mathrm{e}, \mathrm{H}}(r)}{\beta_{\mathrm{p}, \mathrm{ch}}} \simeq 1.65 \times 10^{2}$

meaning that electron impact of $\mathrm{H}$-atoms compared to charge exchange in the region downstream of the shock is the highly dominant loss process for incoming interstellar $\mathrm{H}$-atoms. 


\section{Conclusions}

We have shown in this paper that, contrary to the hitherto conventional thinking, solar wind electrons cannot be expected to keep thermal equilibrium with solar wind protons, at least not after passage of the solar wind plasma over the termination shock. Due to their interactions with the shock-associated electric field, they undergo a substantial heating process which in its magnitude depends on the shock compression ratio $s$ and on the bulk velocity difference $\Delta U_{1}$ at the shock. It does, however, practically not depend on the strength and direction of the magnetic field at the shock surface, since the Bunemann instability redistributing kinetic overshoot velocities into thermal electron speeds operates so strongly that electrons essentially do not recognize the background magnetic field before becoming thermalized. This then also indicates something concerning the global interaction of solar wind electrons when passing over the 3-dimensional geometry of the shock surface. One may conclude that the downstream electron temperature only varies through variable upstream solar wind bulk velocities and through correspondingly variable compression ratios $s$. Furthermore, it may now be plausible that the $10^{6} \mathrm{~K}$ hot downstream electrons do spatially diffuse relative to the background solar wind bulk flow and thus behave similar to cosmic ray electrons, as described by Ferreira et al. (2001a, b) and Lange et al. (2006) and in fact measured by Voyager1/-2 (Webber, 2006).

It must of course be stated here that the solar wind termination shock is a multifluid shock phenomenon, where more than just solar wind electrons and protons are involved. Especially the so-called pick-up ions definitely play an important role (see, e.g. Fahr and Chalov, 2008; Zank et al., 2010; Siewert et al., 2013). They collectively as a multifluid plasma define the consistent compression ratio as shown in the implicit multifluid compression equation $s=S(s)$, as derived by Fahr et al. (2012). Instead of making everything consistent, in this paper here we have derived an expression only for the downstream solar wind electron pressure as a function of the compression ratio $s$. In other papers (Chalov and Fahr, 2011, 2013) we have solved the multifluid MHD RankineHugoniot relations; however, there we have parameterized only the downstream electron pressure as a multiple of the downstream solar wind proton pressure.

In these multifluid solutions it is shown that, in fact due to conservation of their higher upstream magnetic moments, pick-up ions consequently appear on the downstream side as a much more heated ion population compared to normal solar wind protons. They thus also constitute the much larger downstream ion pressure compared to normal solar wind protons. Nevertheless it can be shown (Chalov and Fahr, 2013) that these multifluid approaches only then can represent the shock results, i.e. downstream proton temperature and compression ratio (obtained by Voyager- 2 at its termination shock crossing), if the downstream electron temperature is higher than the downstream solar wind proton temperature by about a factor 10 to 15 (see Chalov and Fahr, 2013). This factor we do in fact obtain with our present calculations.

Finally we should confess here that the problem of thermalizing the shock-electrons is not as clear-cut as we treat it in this paper. We only consider the Bunemann instability and can show that under cold plasma conditions and low electron beta conditions this instability can do the job of efficiently thermalizing the electron overshoot kinetic into electron thermal energy. For this process to operate one should consider the foot region of the shock as a place of electron heating where the background plasma can be considered as sufficiently cold. We think that in fact the ion heating takes place further downstream in connection with ion pitch-angle scattering. As shown by Scholer and Matsukiyo (2004), in competition to the Bunemann instability also the modified twostream instability should be considered as effectively heating electrons. The relative efficiency of these two instabilities is strongly related to the Mach number of the shock (i.e. below or above whistler critical Mach numbers) and of the upstream electron plasma beta value. For electron plasma beta values below 0.02 the Bunemann instability seems to dominate, and we believe that we are in that region of low electron plasma beta values.

Acknowledgements. Hans Fahr is grateful to the International Space Science Institute (ISSI) at Bern, Switzerland, for inviting him to the workshop "The Heliopause" organised by R. Jokipii.

This work was partially supported by the Russian Academy of Sciences program "Fundamental problems of research and exploration of the solar system" and partially by the Russian-German bi-national cooperation grant: 436 RUS 113/110/0-4 sponsored by the Deutsche Forschungsgemeinschaft DFG and the Russian Science Foundation RFFI.

Topical Editor C. Owen thanks two anonymous referees for their help in evaluating this paper.

\section{References}

Alexandrov, A. F., Bogdankevich, L. S., and Rukhadze, A. A.: Principles of plasma electrodynamics, Spinger Series in Electrophysics, Springer Verlag, Berlin, vol. 9, 488 p., 1984.

Chalov, S. V. and Fahr, H. J.: Spatial variation of the supersonic thermal plasma flow downstream of the termination shock, Adv. Space Res., 47, 1523-1528, 2011.

Chalov, S. V. and Fahr, H. J.: The role of solar wind electrons at the solar wind termination shock, Monthly Notices Royal Astron. Soc., 433, L40-L45, 2013.

Chen, F. F.: Plasma physics and controlled fusion, Plasma Physics, Plenum Press, NY, vol. 1, 421 p., 1984.

Fahr, H. J. and Chalov, S. V.: Supersonic solar wind ion flows downstream of the termination shock explained by a two-fluid shock model, Astron. Astrophys., 490, L35-L38, 2008.

Fahr, H. J. and Siewert, M.: Revisiting the role of magnetic moments in heliospheric plasmas, Astron. Astrophys., 552, A38, 1$11,2013$. 
Fahr, H. J., Siewert, M., and Chashei, I. V.: Phasespace transport of a quasi-neutral multifluid plasma over the solar wind MHD termination shock, Astrophys. Space Sci., 341, 265-276, 2012.

Ferreira, S. E. S., Potgieter, M. S., Burger, R. A., Heber, B., and Fichtner, H.: Modulation of Jovian and galactic electrons in the heliosphere; I. Latitudinal transport of a few MEV electrons, J. Geophys. Res., 106, 24979-24987, 2001a.

Ferreira, S. E. S., Potgieter, M., Burger, R., Heber, B., and Fichtner, H.: Modulation of Jovian and Galactioc electrons; II. radial transport of a few MEV electrons, J. Geophys. Res., 106, 2931329318, $2001 b$.

Fichtner, H., Sreenivasan, S. R., and Fahr, H. J.: Cosmic ray modulation and a non-spherical heliospheric shock, Astro. Astrophys., 308, 248-260, 1996.

Kissmann, R., Fichtner, H., Heber, B., Ferreira, S. E. S., and Potgieter, M. S.: First results of a new 3-D model of the timedependent modulation of electrons in the heliosphere, Adv. Space Res., 32, 681-686, 2003.

Lange, D., Fichtner, H., and Kissmann, R.: Time-dependent 3D modulation of Jovian electrons, Astron. Astrophys., 449, 401410, 2006.

Langner, U. W., de Jager, O. C., and Potgieter, M. S.: On the local interstellar spectrum for cosmic ray electrons, Adv. Space Res., 27, 517-522, 2001.

Leroy, M. M., Winske, D., Goodrich, C. C., Wu, C. S., and Papadopoulos, K.: Structure of perpendicular bow shocks, J. Geophys. Res., 87, 5081-5093, 1982

Lotz, W.: Electron-Impact Ionization Cross-Sections and Ionization Rate Coefficients for Atoms and Ions, Astrophys. J. Supplement, 14, p. 207, 1967.

Nkosi, G. S., Potgieter, M. S., and Webber, W. R.: Modelling of lowenergy galactic electrons in the heliosheath, Adv. Space Res., 48, 1480-1489, 2011.

Parker, E. N.: The passage of energetic charged particles through interplanetary space, Planet. Space Sci., 13, 9-49, 1965.

Potgieter, M. S.: Heliospheric modulation of galactic electrons: Consequences of new calculations for the mean free path of electrons between $1 \mathrm{MeV}$ and $\sim 10 \mathrm{GeV}$, J. Geophys. Res., 101, 24411-24422, 1996.

Potgieter, M. S. and Nndanganeni, R. R.: The solar modulation of electrons in the heliosphere, Astrophys. Space Sci., 345, 33-40, 2013.

Richardson, J. D., Kasper, J. C., Wang, C., Belcher, J. W., and Lazarus, A. J.: Cool heliosheath plasma and deceleration of the upstream solar wind at the termination shock, Nature, 454, 63$66,2008$.
Rucinski, D. and Fahr, H. J.: The influence of electron impact ionizations on the distribution of interstellar helium in the inner heliosphere: possible consequences for the determination of the interstellar helium parameters, Astron. Astrophys., 224, 290-298, 1989.

Sarris, E. T. and Van Allen, J. A.: Effects of interplanetary shock waves on energetic charged particles, J. Geophys.Res., 79, 41574173, 1974.

Scholer, M. and Matsukiyo, S.: Nonstationarity of quasiperpendicular shocks: a comparison of full particle simulations with different ion to electron mass ratio, Ann. Geophys., 22, 2345-2353, doi:10.5194/angeo-22-2345-2004, 2004.

Schwartz, S. J., Thomsen, M. F., Bame, S. J., and Stansberry, J.: Electron heating and the potential jump across fast mode shocks, J. Geophys. Res., 93, 12923-12931, 1988.

Sgro, A. G. and Nielson, C. W.: Hybrid model studies of ion dynamics and magnetic field diffusion during pinch implosions, Phys. Fluids, 19, 126-138, 1976.

Siewert, M., Fahr, H. J., McComas, D. J., and Schwadron, N. A.: Spectral properties of $\mathrm{keV}$-energetic ion populations inside the heliopause reflected by IBEX-relevant energetic neutral atoms, Astron. Astrophys., 551, 1-13, 2013.

Terasawa, T.: Energy spectrum and pitch angle distribution of particles reflected by MHD shock waves of fast mode, Planet. Space Sci., 27, 193-201, 1979.

Tokar, R. L., Aldrich, C. H., Forslund, D. W., and Quest, K. B.: Nonadiabatic electron heating at high-Mach-number perpendicular shocks, Phys. Rev. Lett., 56, 1059-1062, 1986.

Verscharen, D. and Fahr, H. J.: A kinetic description of the dissipative quasi-parallel solar wind termination shock, Astron. Astrophys., 487, 723-729, 2008.

Webber, W. R.: Modulation in the heliosheath and beyond, in: Physics of the Inner Heliosheath, CP 858, pp. 135-140, edited by: Heerikhuisen, J., Florinski, V., Zank, G. P., and Pogorelov, N., American Institute of Physics, 2006.

Wu, P., Winske, D., Gary, S. P., Schwadron, N. A., and Lee, M. A.: Energy dissipation and ion heating at the heliospheric termination shock, J. Geophys. Res., 114, A08103, 1-15, 2009.

Zank, G. P., Heerikhuisen, J., Pogorelov, N. V., Burrows, R., and McComas, D.: Microstructure of the Heliospheric Termination Shock: Implications for Energetic Neutral Atom Observations, The Astrophys. J., 708, 1092-1106, 2010. 